\title{
Fusion and breakup of halo nuclei
}

\author{
M.S. Hussein ${ }^{a}$, L.F. Canto $^{b}$ and R. Donangelo ${ }^{b}$ \\ ${ }^{a}$ Instituto de Física, Universidade de São Paulo, \\ C.P. 66318, 05389-970, São Paulo, SP, Brazil \\ ${ }^{b}$ Instituto de Física, Universidade Federal do Rio de Janeiro, \\ C.P. 68528, 21945-970, Rio de Janeiro, RJ, Brazil
}

We discuss the effect of the coupling to the break-up channel on the total fusion involving a halo nucleus with a heavy target. We show that there is a competition between the hindrance arising from this coupling mostly at above barrier energies, and the enhancement which ensues at sub-barrier energies owing to the static effect of the extended matter distribution and the coupling to the soft modes.

\section{INTRODUCTION}

The fusion of heavy ions is of paramount importance in astrophysics and in the production of super heavy elements (SHE). With the advent of secondary beams of neutron and proton rich nuclei, it is important to assess how the complete fusion of these nuclear species behave as a function of bombarding energy especially near the Coulomb barrier [1]. Several of the light neutron and proton rich nuclei exhibit halo structures, with a compact core plus one or two loosely bound nucleons occupying a far away orbit. Systems such is ${ }^{11} \mathrm{Li}$ and ${ }^{6} \mathrm{He}$ are two-neutron, borromean halo nuclei, while ${ }^{11} \mathrm{Be}$ and ${ }^{19} \mathrm{C}$ are one-neutron halo nuclei. The isotope ${ }^{8} B$ has been confirmed to be a one-proton halo while ${ }^{17} \mathrm{~F}$ is a normal nucleus in its ground state but acquires a one-proton halo nature in its 1st excited state. We ask the question of how the above systems fuse. One important feature to remember about these loosely bound systems is their collective response. They exhibit the so-called soft giant resonances (pygmy resonances), the most notorious of which is the soft dipole resonance, very nicely confirmed in ${ }^{6} \mathrm{He}$ by Nakayama et al [2]. On the other hand the threshold for break-up is very small $(<1 \mathrm{MeV})$, making the study of the fusion of these nuclei with heavy targets an interesting endeavour, since the coupling to the soft modes tend to enhance fusion while the coupling to break-up reduces fusion. The latter is true since, if the Coulomb and nuclear coupling is of long range and strong, which is the case when fusion occurs with a heavy nucleus, then the projectile (the halo nucleus) would break before it reaches the target. This led us to propose a model for complete fusion which looks like

$$
\sigma_{C F}=\frac{\pi}{k^{2}} \sum_{i}(2 \ell+1) T_{\ell, i}(E) P_{\ell, i}(E)
$$


where $T_{\ell, i}(E)$ is the fusion transmission coefficient for the $l-t h$ partial wave and $P_{\ell, i}(E)$ is the break-up survival probability $[3,4,5]$. The sum in Eq.(1) is over all bound states in the projectile and target. Simple approximations were then used for $T_{\ell, i}(E)$ and $P_{\ell, i}(E)$ and a few examples were considered. The overall result from this model was a reduction of $\sigma_{C F}$ near and above the barrier energy and an enhancement below. This trend was later confirmed by Takigawa et al [6] and more recently by the coupled channel calculation with continuum discretization of Hagino et al [7].

Though our results were based on intuitive arguments, one can derive Eq. (1) from general reaction theory. The same theory [8] allows the obtention of the incomplete fusion cross-section $\sigma_{I C F}$, which in many instances is not easy to distinguish from $\sigma_{C F}$. As an example we take the fusion of ${ }^{6} \mathrm{He}$ recently measured by Trotta et al [9] on ${ }^{238} \mathrm{U}$ and Kolata et al [10] on ${ }^{209} \mathrm{Bi}$. Here the incomplete fusion is that where ${ }^{6} \mathrm{He}$ is broken up and ${ }^{5} \mathrm{He}$ or ${ }^{4} \mathrm{He}$ is absorbed by the target. The remaining neutrons when detected are difficult to distinguish from evaporation neutrons from the compound nucleus.

In the present contributions we give a brief review of theoretical attempts to calculate the fusion of halo nuclei with heavy targets. We also present a short review of the experimental data currently available.

\section{EXPERIMENTAL DATA}

So far, four measurements were made on the fusion of ${ }^{11} \mathrm{Be}$ and ${ }^{6} \mathrm{He}$ with heavy targets at near-barrier energies. Signorini et al [11] reported the measurements of the fusion systems ${ }^{9,10,11} \mathrm{Be}+{ }^{209} \mathrm{Bi}$ at energies close to the Coulomb barrier. These authors reach the conclusion that in the weakly bound normal nucleus ${ }^{9} \mathrm{Be}$, the fusion with ${ }^{209} \mathrm{Bi}$ is found significantly reduced at above barrier energies, owing to the coupling to the breakup channel. This supports our discussion in the introduction concerning the irreversible nature of the coupling to the break-up continuum. The cases of ${ }^{10} \mathrm{Be}$ and ${ }^{11} \mathrm{Be}$ are more subtle to understand. However, the more recent results of [9] on ${ }^{6} \mathrm{He}+{ }^{238} \mathrm{U}$ and [10] on ${ }^{6} \mathrm{He}+{ }^{209} \mathrm{Bi}$ do indicate that at below the barrier energies the fusion cross section is enhanced. Thus the halo shows itself as enhancement at sub-barrier energies and the break-up hinders the fusion; the effect becoming more important at above barrier energies. We should of course remind the readers that ${ }^{6} \mathrm{He}$ is a borromean 2n-halo nucleus while ${ }^{11} \mathrm{Be}$ is one-neutron halo nucleus. Signorini at al [11] found that the, normal, strongly bound ${ }^{10} \mathrm{Be}$ isotope presents a larger fusion cross section than that of ${ }^{11} \mathrm{Be}$ at sub-barrier energies. This effect does not fit into the picture we have just presented. We should mention that is the case of the comparison between ${ }^{6} \mathrm{He}$ and ${ }^{4} \mathrm{He}$ fusion with ${ }^{209} \mathrm{Bi}$ by Kolata et al [10], the effects of the halo (enhancement) at sub-barrier energies, were quite evident.

The fusion of the proton rich isotope ${ }^{17} \mathrm{~F}$ with ${ }^{208} \mathrm{~Pb}$ was measured by Rehm et al [12]. This weakly bound nucleus has a normal ground state, but its first excited state is mostly $\ell=0$ and seems to exhibit halo features. The results of Ref. (12) indicate a rather normal behaviour of the complete fusion cross-section, with a very small effect due 
to break-up, though the break-up channel coupling $\left({ }^{17} F \rightleftarrows{ }^{16} O+p\right)$ would seem to reduce the Coulomb barrier, contrary to the case of the fusion of neutron-rich, drip-line, nuclei.

If there is strong break-up effect on the fusion cross-section that leads to its reduction then the break-up cross-section itself should be noticeable. In the recent measurement by Hinde et al [13], of ${ }^{9} \mathrm{Be}+{ }^{208} \mathrm{~Pb}$ at near-barrier energies the elastic break-up cross-section was measured. Here the prompt break-up channel is $\alpha+\alpha+n$ with a $Q=1.57 \mathrm{MeV}$. The authors reach the conclusion that this channel is responsible for the large suppression of the complete fusion cross-section at above-barrier energies (one-dimensional barrier calculation has to be multiplied by 0.68 to account for the observation).

This reduction in the complete fusion seems to be accompanied by a large incomplete fusion cross-section where one of the $\alpha$-particles fuses with ${ }^{208} \mathrm{~Pb}$. Hinde et al [15] found that the incomplete fusion probability is about $0.32 \pm 0.07$, clearly attesting for the unitarity constraint that $P_{C F}+P_{I C F}$ should be one. The corresponding total fusion cross-section $\sigma_{F} \equiv \sigma_{C F}+\sigma_{I C F}$ should be accountable by the simple one-dimensional barrier penetration model. These findings corroborates the earlier results of Dasgupta et al [14] which showed a considerable hindrance in $\sigma_{F}$ at above barrier energies. Hinde et al [13] also discuss the dependence of the fusion hindrance factor on the charge of the target. They found that $P_{C F}$ depends strongly on the target charge. In the experiment of Ref. [15] involving ${ }^{6,7} \mathrm{Li}$ fusion with ${ }^{9} \mathrm{Be}$ and ${ }^{12} \mathrm{C}$ targets, it was reported that suppression of up to $70 \%$ is found. This, however, was contested recently $[16,17]$ through independent measurements of several of the systems studied in Ref. [15]. The conclusions of $[16,17]$ is that there is no supression of $\sigma_{C F}$ for these light systems.

In fact, the $70 \%$ fusion hindrance in the fusion of ${ }^{6} \mathrm{Li}$ and ${ }^{7} \mathrm{Li}$, was indeed found in Ref. [18] but on a heavy target, ${ }^{209} \mathrm{Bi}$. We can use Eq. (1) as a guide and take for the break-up survival probability the simple DPP form [4] with the Coulomb DPP proportional to $Z_{T}^{2}$ $[19,20]$. If the effect of the survival probability on the fusion of ${ }^{7} \mathrm{Li}+{ }^{209} \mathrm{Bi}$ is $75 \%$ [18] at near-barrier energies, then, for the ${ }^{7} \mathrm{Li}+{ }^{12} \mathrm{C}$ case of [15]. the effect is tremendously reduced since the quantity $\left(Z_{C} / Z_{B i}\right)^{2} \simeq 0.005$, implies an over all reduction in $\sigma_{C F}$ for ${ }^{7} \mathrm{Li}+{ }^{12} \mathrm{C}$ of about $\exp (-0.005 \ln (1 / 0.75))=0.99989$, namely no reduction at all. Of course there are the nuclear break-up effects, but these are very small as well. Accordingly there is no suppression of $\sigma_{C F}$ for light systems, in total agreement with the conclusion of Refs. $[16,17]$.

Of course other processes such as fast fission, also results in a considerable hindrance in the complete fusion involving heavy targets [21]. This is similar to the effects of Deep Inelastic Collision on $\sigma_{C F}$ in the so-called Region II [1].

\section{THEORETICAL MODELS}

Theoretical description of tunnelling phenomena, such as sub-barrier fusion, in the case of strong channel coupling is made with recourse to coupled channels theory [22]. This theory has been extensively and successfully used to describe the sub-barrier fusion of stable nuclei, which invariably exhibits enhancement over the simple one-dimensional barrier penetration model [1]. The channels that are taken into account correspond to bound states of the partners. In extending this picture to the fusion of halo nuclei, one must take into account the break-up channel coupling. This has been done is the past for deuteron scattering from different targets within the so-called Continuum Discretized 
Coupled Channels (CDCC) Method [23]. The effect of the deuteron break-up on the elastic scattering was found to be representable by a weaker attraction, and absorption (implying that the dynamic polarization potential, DPP, associated with the deuteron break-up has a repulsive real part and attractive imaginary part (absorption) $[4,5,23,24]$ ). It was from these findings that we were lead to use Eq. (1) $[4,5,24]$ which later modified to incorporate the real part of the DPP $[3,24]$ in the survival probability, $P_{\ell, i}(E)$. In a later publication, Dasso and Vitturi [25] used an effective one bound "break-up" channel and reached the conclusion that the break-up leads to enhancement of fusion. In a later paper, these authors [7] modified their work by adding, many bound "break-up" channels and reached a similar conclusion as ours, namely, break-up hinders fusion at above-barrier energies and enhances it at lower energies. A more quantitative comparison shows that the shape of their cross section is somewhat different from ours at the barrier region and the transition from enhancement to hindrance occurs at a slightly higher energy.

In fact, the effect of the widths of these resonances (excited states with finite life times) was studied in Refs. [26,27]. The overall effect of the width as compared to bound excited state, is a reduction in fusion. However, at very low energies, the pygmy resonances act as if they were bound excited states and thus the enhancement seen in the sub-barrier fusion calculation of Ref. [7] must arise from the above effects, as well as the farther extension of the matter distribution, and not to break-up.

Quite recently, A. Diaz-Torres and I.J. Thompson [28] performed the most complete calculation of the fusion cross-section of halo nuclei using the CDCC method. They found a large reduction in the complete fusion both above and below the barrier for the system ${ }^{11} \mathrm{Be}+{ }^{208} \mathrm{~Pb}$. They also calculated the incomplete fusion cross-section as that corresponding to flux loss from the break-up channels (the continuum is discretized into several channels). This definition of $\sigma_{I C F}$ which follows that of Hagino et al [7] is an overestimate since the incomplete fusion is the fusion of the heavy charged fragment only. See Ref. [8], for more details. In fact in many of the papers found in the literature on fusion of two-cluster (or three-cluster) nuclei, there is always ambiguities in actually measuring and also calculating $\sigma_{I C F}$. An exception to this is found in the work of Hinde et al [13] where $\sigma_{I C F}$ was correctly measured for the system ${ }^{11} \mathrm{Be}+{ }^{209} \mathrm{Bi}$.

It is of interest to analyze the general structure of the total reaction cross section, $\sigma_{R}$, of the system studied by A. Diaz-Torres and Thompson [28], namely ${ }^{11} \mathrm{Be}+{ }^{208} \mathrm{~Pb}$ at low energies. From the formal analysis of Hussein [29], we can write the following expression for the reaction cross-sector

$$
\sigma_{R}=\sigma_{D I R}+\sigma_{F}
$$

where $\sigma_{D I R}$ is the total direct cross section which contains, in the specific case of ${ }^{11} B e+{ }^{208}$ $P b$ at very low energies, just the inelastic, $\sigma_{I N E}$ (mostly Coulomb) excitation of the $1 / 2^{-}$ state at $0.32 \mathrm{MeV}$ and the elastic break-up cross-section corresponding to ${ }^{11} B e$ splitting into ${ }^{10} \mathrm{Be}$ plus a neutron in addition to the situation where the neutron is absorbed by the target forming ${ }^{209} \mathrm{~Pb}$, namely the one-neutron removal cross-section $\sigma_{-n}$. The component $\sigma_{F}$ is the total fusion cross-section alluded to earlier, which contains the complete fusion $\left({ }^{11} \mathrm{Be}+{ }^{208} \mathrm{~Pb} \rightarrow{ }^{219} \mathrm{Rn}\right)$ plus the incomplete fusion $\sigma_{I C F}$ where ${ }^{10} \mathrm{Be}$ is captured by the target It is more likely that $\sigma_{I C F}$ should be just ${ }^{10} \mathrm{Be}+{ }^{208} \mathrm{~Pb} \rightarrow{ }^{218} \mathrm{Rn}$ since ${ }^{10} \mathrm{Be}$ is long-lived in the present context. Thus $\sigma_{I C F}=\sigma_{-}{ }^{10} B e$. 
Accordingly we can write

$$
\sigma_{D I R}=\sigma_{I N E}+\sigma_{b u p}+\sigma_{-n}
$$

and

$$
\sigma_{F}=\sigma_{C F}+\sigma_{-10}{ }^{10} e
$$

In the calculation of Hagino et al [7] and of Diaz-Torres and Thompson [28], the total fusion cross-section, $\sigma_{F}$ was obtained from

$$
" \sigma_{F} "=\sigma_{C F}+\sigma_{-10} B e+\sigma_{-n},
$$

or

$$
" \sigma_{F} " \equiv \sigma_{C F}+" \sigma_{I C F} "
$$

Therefore " $\sigma_{I C F}$ " is an overestimate of the incomplete fusion cross-section. Further, if $\sigma_{D I R}$ is ignored, as done in both references, then unitarity would require an underestimate of $\sigma_{C F}$. This explains the findings of [28] and points to ways of correcting them. One should resort to three - or four - body calculations in order to calculate $\sigma_{-n}$ and $\sigma_{-{ }^{10}} B e$. Short of a full fledged Faddeev (3-bodies) or Jakubovskij (4-bodies) treatments of the reactions of one - and two - nucleon halo nuclei, respectively, one resorts to approximate treatments. In Ref. [8], using the Hussein-McVoy [30] formalism for inclusive breakup reactions and improvements on it, it was find that one may calculate the removal cross-section of the heavy fragment, $\sigma_{-}{ }^{10} B e$, according to the practical formula [8]

$$
\left.\sigma_{I C F}=\sigma_{-{ }_{10} B e}=\frac{\pi}{k_{10_{B e}}^{2}} \frac{v^{\prime}}{v} \sum_{\ell_{1}}\left(2 \ell_{1}+1\right) T_{\ell_{1}}^{(10} B e\right)\left(E_{1}\right)\left[1-P_{\ell_{o}}(E)\right]\left[1-T_{\ell_{2}}^{(n)}\left(E_{2}\right)\right]
$$

where $\left[1-P_{\ell_{o}}(E)\right]$ is the break-up probability of the projectile, $T_{\ell_{1}}^{(10 B e)}\left(E_{1}\right)$ is the fusion probability for ${ }^{10} B e$ and $\left[1-T_{\ell_{2}}^{(n)}\left(E_{2}\right)\right]$ is the survival probability of the neutron in the process ${ }^{11} \mathrm{Be} \rightarrow{ }^{10} \mathrm{Be}+n$. In Eq. (7) $v$ and $v^{\prime}$ are the relative velocities in the elastic and break-up channels. The calculation of $\sigma_{-10} B e$ is presently in progress. The above considerations should be worked out within a few-body treatment of fusion [31]. However, $\sigma_{I C F}$, according to Eq. (7), may be calculated within the CDCC of Ref. [28].

One last comment concerning the complete fusion cross section calculated within CDCC by Diaz-Torres and I.J. Thompson [28] for ${ }^{11} \mathrm{Be}+{ }^{208} \mathrm{~Pb}$. There is a reason to expect another mechanism that would further enhance the very low values of $\sigma_{C F}$. After discretizing the continuum, and thus replacing the density of states of the two-clusters continuum by a much smaller density of discrete states, one looses part of the fusion flux. This lost flux, should be accounted for within CDCC by an appropriate incoherent addition of a fluctuation fusion contribution. Ref. [32] discusses in fact the statistical nature of the coupling to the continuum, which would require, besides the "average" CDCC , the statistical treatment of the fluctuation, just as is done in the description of DIC [33]. This should increase the Diaz-Torres and Thompson [28] complete fusion cross-section. Work along this line is in progress.

\section{CONCLUSIONS}


We have presented in this short review an account of the present status of the activities in the field of the fusion of halo nuclei. We have discuss the results of the available data and critically assessed some of the interpretations. We have also discuss theoretical attempts made so far to calculate the complete and incomplete fusion cross-sections. In particular we went at length in considering the effect of the coupling to the break-up channel. The overall conclusion that seems to emerge from several of these studies is that this coupling always leads to a reduction in $\sigma_{C F}$ above the barrier. At sub-barrier energies, other features of halo nuclei (extended mass distribution, pygmy resonances) come into play and they lead to an enhancement of $\sigma_{C F}$ compared to the single channel model results. We have also analysed the recent calculation of Diaz-Torres and Thompson [28] within the CDCC method and pointed to ways of improving the theory.

Fusion and break-up of halo nuclei continues to be a challenging field and certainly deserves further experimental and theoretical studies.

\section{ACKNOWLEDGMENT}

This work was supported in part by FAPESP and the CNPq.

\section{REFERENCES}

1. For a recent review see, C.A. Bertulani, M.S. Hussein and G. Münzenberg, "Physics of Radioactive Beams" (Nova Science, New York, 2001), Chapter 12.

2. S. Nakayama et al. Phys. Rev. Lett 85 (2002) 262.

3. M.S. Hussein, M.P. Pato, L.F. Canto and R. Donangelo, Phys. Rev. C46 (1992) 377; Phys. Rev. C47 (1993) 2398.

4. L.F. Canto, R. Donangelo, P. Lotti and M.S. Hussein, Phys. Rev. C52 (1995) R2848; L.F. Canto et al. J. Phys. G23 (1997) 1465; W.H.Z. Cárdenas et al., Nucl. Phys. A703 (2002) 673.

5. M.S. Hussein et al., Nucl. Phys. A588 (1995) 85C.

6. N. Takigawa, M Kuratani and H. Sagawa, Phys. Rev. C47 (1993) R2470.

7. K. Hagino, A. Vitturi, C.H. Dasso and S. Lenzi, Phys. Rev. C51 (2000) 037602.

8. L.F. Canto et al., Phys. Rev. C58 (1998) 1107.

9. M. Trotta et al., Phys. Rev. Lett. 84 (2000) 2342.

10. J.J. Kolata et al., Phys. Rev. Lett. 81 (1998) 4580.

11. C. Signorini et al., Eur. Phys. J. A5 (1999) 7; C. Signorini, Eur. Phys. J. A13 (2002) 129.

12. K.E. Rehm et al., Phys. Rev. Lett. 81 (1998) 3341.

13. D.J. Hinde et al., Phys. Rev. Lett. 89 (2002) 272701-1. 
14. M. Dasgupta et al., Phys. Rev. Lett. 82 (1999) 1395.

15. J. Takahashi et al., Phys. Rev. Lett. 78 (1997) 30; A. Szanto de Toledo et al., Nucl. Phys. A679 (2000) 175.

16. A. Mukherjee et al., Nucl. Phys. A635 (1998) 205; A. Mukherjee et al., Nucl. Phys. A645 (1999) 13; A. Mukherjee et al., Phys. Ltett. B526 (2002) 295.

17. R.M. Anjos et al., Phys. Lett. B534 (2002) 45.

18. M. Dasgupta et al., Phys. Rev. C66 (2002) 041602 (R).

19. M.V. Andrés, J. Gómez-Camacho and M.A. Nagarajan, Nucl. Phys. A579 (1994) 273.

20. L.F. Canto, R. Donangelo, P. Lotti and M.S. Hussein, Nucl. Phys. A589 (1995) 117.

21. D.J. Hinde, M. Dasgupta and A. Mukherjee, Phys. Rev. Lett. 89 (2002) 282701.

22. See, e.g. M. Dasgupta et al., Ann. Rev. Nucl. Part. Sci. 48 (1998) 401; G.R. Satchler et al., Ann. Phys. 178 (1987) 110; A.M.S. Breitschaft et al., Ann. Phys. 243 (1995) 420.

23. N. Anstern et al., Phys. Rep. 154 (1987) 110.

24. L.F. Canto et al., Nucl. Phys. A542 (1992) 131.

25. C.H. Dasso and A. Vitturi, Phys. Rev. C50 (1994) R12.

26. M.S. Hussein and A.F.R. de Toledo Piza, Phys. Rev. Lett. 72 (1994) 2693; M.S. Hussein et al., Phys. Rev. C51 (1995) 846.

27. K. Hagino and N. Takigawa, Phys. Rev. C58 (1998) 2872.

28. A. Diaz-Torres and I.J. Thompson, Phys. Rev. C65 (2002) 024606.

29. M.S. Hussein, Phys. Rev. C30 (1984) 962.

30. M.S. Hussein and K. W. McVoy, Nucl. Phys. A445 (1985) 124.

31. B.V. Carlson, T. Frederico and M.S. Hussein, to be published.

32. S. Drozdz, J. Okolowicz, M. Polszajazak and I. Rooter, Phys. Rev. C62 (2000) 024313.

33. See, e.g. D. Agassi, C.M. Ko and H.A. Weidenmüller, Ann. Phys. (N.Y.) 107 (1977) 140; H. Feshbach, A. K. Kerman and S.E. Koonin, Ann. Phys. (N.Y.) 125 (1980) 429. 